\title{
Tridiagonal Symmetries of Models of Nonequilibrium Physics ${ }^{\star}$
}

\author{
Boyka ANEVA
}

Institute for Nuclear Research and Nuclear Energy, Bulgarian Academy of Sciences, 72 Tzarigradsko chaussee, 1784 Sof ia, Bulgaria

E-mail: blan@inrne.bas.bg

Received March 03, 2008, in final form July 14, 2008; Published online July 28, 2008

Original article is available at http://www.emis.de/journals/SIGMA/2008/056/

\begin{abstract}
We study the boundary symmetries of models of nonequilibrium physics where the steady state behaviour strongly depends on the boundary rates. Within the matrix product state approach to many-body systems the physics is described in terms of matrices defining a noncommutative space with a quantum group symmetry. Boundary processes lead to a reduction of the bulk symmetry. We argue that the boundary operators of an interacting system with simple exclusion generate a tridiagonal algebra whose irreducible representations are expressed in terms of the Askey-Wilson polynomials. We show that the boundary algebras of the symmetric and the totally asymmetric processes are the proper limits of the partially asymmetric ones. In all three type of processes the tridiagonal algebra arises as a symmetry of the boundary problem and allows for the exact solvability of the model.
\end{abstract}

Key words: driven many-body systems; nonequilibrium; tridiagonal algebra; Askey-Wilson polynomials

2000 Mathematics Subject Classification: 82C10; 60J60; 17B80

\section{Introduction}

Out of the rich variety of phenomena in nature the most interesting occur in nonequilibrium conditions and their complex behaviour is far from being well understood. The study of nonequilibrium phenomena is of current interest and the way to describe the general characteristics of a system out of equilibrium goes through the analysis of mathematical models. Such models have to be simple enough, still they must be physically significant to exhibit the structure of the complex phenomena. Stochastic interacting particle systems $[1,2,3]$ received a lot of attention. Among these, the asymmetric simple exclusion process (ASEP) has become a paradigm in nonequilibrium physics due to its simplicity, rich behaviour and wide range of applicability. Introduced originally as a simplified model of one dimensional transport for phenomena like kinetics of biopolymerization [6], it has found applications from traffic flow [4, 5], to interface growth [7], shock formation, hydrodynamic systems obeying the noisy Burger equation, problems of sequence alignment in biology [8].

At large time the ASEP exhibits relaxation to a steady state, and even after the relaxation it has a nonvanishing current. An intriguing feature is the occurrence of boundary induced phase transitions [2] and the fact that the bulk properties depend strongly on the boundary rates.

The asymmetric exclusion process is an exactly solvable model of a lattice diffusion system of particles interacting with a hard core exclusion, i.e. the lattice site can be either empty or occupied by a particle. As a stochastic process it is described in terms of a probability

\footnotetext{
*This paper is a contribution to the Proceedings of the Seventh International Conference "Symmetry in Nonlinear Mathematical Physics" (June 24-30, 2007, Kyiv, Ukraine). The full collection is available at http://www.emis.de/journals/SIGMA/symmetry2007.html
} 
distribution $P\left(s_{m}, t\right)$ of a stochastic variable $s_{m}=0,1$ at a site $m=1,2, \ldots, L$ of a linear chain. A state on the lattice at a time $t$ is determined by the set of occupation numbers $s_{1}, s_{2}, \ldots, s_{L}$ and a transition to another configuration $s^{\prime}$ during an infinitesimal time step $d t$ is given by the probability $\Gamma\left(s, s^{\prime}\right) d t$. With the restriction of dynamics that changes of configuration can only occur at two adjacent sites, the rates for such changes depend on these sites only. The two-site rates $\Gamma\left(s, s^{\prime}\right) \equiv \Gamma_{s_{m}^{\prime}, s_{m+1}^{\prime}}^{s_{m}, s_{m+1}}=\Gamma_{j l}^{i k}, i, j, k, l=0,1$ are assumed to be independent from the position in the bulk. Due to probability conservation $\Gamma(s, s)=-\sum_{s^{\prime} \neq s} \Gamma\left(s^{\prime}, s\right)$. For diffusion processes the transition rate matrix becomes simply $\Gamma_{k i}^{i k}=g_{i k}$. At the boundaries, i.e. sites 1 and $L$ additional processes can take place with rates $L_{i}^{j}$ and $R_{i}^{j}(i, j=0,1)$. In the set of occupation numbers $\left(s_{1}, s_{2}, \ldots, s_{L}\right)$ specifying a configuration of the system $s_{i}=0$ if a site $i$ is empty, $s_{i}=1$ if there is a particle at a site $i$. Particles hop to the left with probability $g_{01} d t$ and to the right with probability $g_{10} d t$. The event of exchange happens if out of two adjacent sites one is a vacancy and the other is occupied by a particle. The symmetric simple exclusion process is known as the lattice gas model of particle hopping between nearest-neighbour sites with a constant rate $g_{01}=g_{10}=g$. The partially asymmetric simple exclusion process with hopping in a preferred direction is the driven diffusive lattice gas of particles moving under the action of an external field. The process is totally asymmetric if all jumps occur in one direction only, and partially asymmetric if there is a different non-zero probability of both left and right hopping. The number of particles in the bulk is conserved and this is the case of periodic boundary conditions. In the case of open systems, the lattice gas is coupled to external reservoirs of particles of fixed density. The most interesting examples (see [9] and references therein for a review) are phase transitions inducing boundary processes $[2,10]$ when a particle is added with probability $\alpha d t$ and/or removed with probability $\gamma d t$ at the left end of the chain, and it is removed with probability $\beta d t$ and/or added with probability $\delta d t$ at the right end of the chain.

The time evolution of the model is governed by the master equation for the probability distribution of the stochastic system $\frac{d P(s, t)}{d t}=\sum_{s^{\prime}} \Gamma\left(s, s^{\prime}\right) P\left(s^{\prime}, t\right)$. It can be mapped to a Schrödinger equation in imaginary time for a quantum Hamiltonian with nearest-neighbour interaction in the bulk and single-site boundary terms $\frac{d P(t)}{d t}=-H P(t)$, where $H=\sum_{j} H_{j, j+1}+H^{(L)}+H^{(R)}$ and the ground state of the Hamiltonian, in general non-Hermitian, corresponds to the steady state of the stochastic dynamics where all probabilities are stationary. The mapping $\Gamma=$ $-\sqrt{q} U_{\mu}^{-1} H_{X X Z} U_{\mu}$, where

$$
U_{\mu}=\bigotimes_{i=1}^{L}\left(\begin{array}{cc}
1 & 0 \\
0 & \mu(\sqrt{q})^{i-1}
\end{array}\right)
$$

(see [11] and [12] for the details) provides a connection to the integrable $S U_{q}(2)$-symmetric $X X Z$ quantum spin chain with $q=\frac{g_{01}}{g_{10}} ; H_{X X Z}$ is the Hamiltonian of the $U_{q}(s u(2))$ invariant quantum spin chain $H_{X X Z}^{Q G r}[13]$ with anisotropy $\Delta_{q}$ and with added non diagonal boundary terms $B_{1}$ and $B_{L}$

$$
\begin{aligned}
H_{X X Z} & =H_{X X Z}^{Q G r}+B_{1}+B_{L} \\
& =-1 / 2 \sum_{i=1}^{L-1}\left(\sigma_{i}^{x} \sigma_{i+1}^{x}+\sigma_{i}^{y} \sigma_{i+1}^{y}-\Delta_{q} \sigma_{i}^{z} \sigma_{i+1}^{z}+h\left(\sigma_{i+1}^{z}-\sigma_{i}^{z}\right)+\Delta_{q}\right)+B_{1}+B_{L} .
\end{aligned}
$$

The transition rates of the ASEP are related to the parameters $\Delta_{q}$ and $h$, and the boundary terms in the following way ( $\mu$ is free parameter, irrelevant for the spectrum)

$$
\Delta_{q}=-1 / 2\left(q^{1 / 2}+q^{-1 / 2}\right), \quad h=1 / 2\left(q^{1 / 2}-q^{-1 / 2}\right),
$$




$$
\begin{aligned}
& B_{1}=\frac{1}{2 \sqrt{q}}\left(\alpha+\gamma+(\alpha-\gamma) \sigma_{1}^{z}-2 \alpha \mu \sigma_{1}^{-}-2 \gamma \mu^{-1} \sigma_{1}^{+}\right) \\
& B_{L}=\frac{\left(\beta+\delta-(\beta-\delta) \sigma_{L}^{z}-2 \delta \mu q^{L / 2-1 / 2} \sigma_{L}^{-}-2 \beta \mu^{-1} q^{-L / 2+1 / 2} \sigma_{L}^{+}\right)}{2 \sqrt{q}} .
\end{aligned}
$$

For nonequilibrium systems, as opposed to the ones in equilibrium, the boundary conditions are of major importance. Emphasizing the dependence of the steady state behaviour on the boundary rates we study tridiagonal algebras of the simple exclusion processes which reveal deep algebraic properties of the latter. In a recent paper [14] we have shown that the boundary symmetry of the open partially asymmetric exclusion process is an Askey-Wilson algebra, the coideal subalgebra of $U_{q}(\hat{s u}(2))$, which allows for the exact solvability in the stationary state. From the boundary AW algebra the tridiagonal boundary algebra of the ASEP follows through the natural homomorphism. The particular values of the structure constants determine the bulk tridiagonal algebra of the ASEP. It is generated by the matrices $D_{0}$ and $D_{1}$ of the matrix product ansatz and the defining relations have the form of the level zero $U_{q}(\hat{s u}(2)) q$-Serre relations. This suggests a framework for analysis of the asymmetric exclusion process. Namely, given the $U_{q}(\hat{s u}(2)) R$-matrix operator $R\left(z_{1} / z_{2}\right) \in \operatorname{End}_{\mathbf{C}} V_{z_{1}} \otimes V_{z_{2}}$, where $V_{z}$ is the two-dimensional $U_{q}(\hat{s u}(2))$ evaluation module, satisfying the Yang-Baxter equation

$$
R_{12}\left(z_{1} / z_{2}\right) R_{13}\left(z_{1}\right) R_{23}\left(z_{2}\right)=R_{23}\left(z_{2}\right) R_{13}\left(z_{1}\right) R_{12}\left(z_{1} / z_{2}\right),
$$

then the transition matrix $\Gamma$ (or equivalently the Hamiltonian) is written as $H=\sum H_{i i+1}$, where the two-site density is obtained as

$$
H_{i i+1}=\left.\frac{d}{d u} P R_{i i+1}\right|_{u=0}
$$

with $P$ the permutation operator and $z_{1} / z_{2}=e^{u}$. The generators act on the quantum space by means of the infinite coproduct and the invariance with respect to the affine $U_{q}(\hat{s u}(2))$ manifests in the property

$$
\left[H, \Delta^{\infty}\left(G_{k}\right)\right]=0
$$

for any of the generators $G_{k}$ of $U_{q}(\hat{s u}(2))$. If we introduce for finite chain a boundary of a particular form, such as diagonal boundary terms, the symmetry is reduced to $U_{q}(s u(2))$ and the invariant Hamiltonian [13] $H_{X X Z}^{Q G r}$ is known [12] to describe a bulk diffusive system with reflecting boundaries. In the presence of a general boundary the symmetry is further reduced to a boundary symmetry whose generators are constructed as linear covariant objects with respect to the bulk quantum group. It turns out that the boundary symmetry of the asymmetric exclusion process is the AW algebra whose elements possess the coproduct properties of two-sided coideals of the bulk quantum $U_{q}(s u(2))$ symmetry. We have implemented [14] the generators of the AW algebra to construct an operator valued $K$-matrix, a solution to the spectral dependent boundary Yang-Baxter equation, also known as a reflection equation

$$
R\left(z_{1} / z_{2}\right)\left(K\left(z_{1}\right) \otimes I\right) R\left(z_{1} z_{2}\right)\left(I \otimes K\left(z_{2}\right)\right)-\left(I \otimes K\left(z_{2}\right)\right) R\left(z_{1} z_{2}\right)\left(K\left(z_{1}\right) \otimes I\right) R\left(z_{1} / z_{2}\right)=0 .
$$

The $K(z)$ matrix is the basic ingredient of the inverse scattering method [15] and such a connection points out to exact solvability beyond the stationary state and description of the dynamics of the process. This can be achieved by applying the technique of the quantum inverse scattering method, and in particular the underlying Bethe Ansatz method to exactly calculate some quantities of physical interest, such as the common spectrum of the commuting conserved quantities.

In the following we first review the algebra of the asymmetric exclusion process, both the boundary and the bulk one. The boundary AW algebra is naturally mapped to a tridiagonal 
algebra whose irreducible infinite-dimensional modules are the AW polynomials as well. We study the $q=1$ limit of the tridiagonal algebra and show that it appears to be the boundary symmetry of the symmetric exclusion process. The structure constants of the tridiagonal boundary algebra are given in terms of the parameters of the symmetric exclusion process. From the boundary algebra, analogously to the case of the ASEP, one obtains, for particular values of the structure constants, the bulk tridiagonal algebra of the symmetric process. We consider the Askey-Wilson algebra of the totally asymmetric exclusion process which can be viewed as a particular $q=0$ limit of the Askey-Wilson algebra. The consequences of these symmetry properties for the exact solvability of the simple exclusion model in the stationary state within the matrix product approach are discussed.

\section{The model within the matrix product approach}

The matrix product approach (MPA) was developed with the aim to describe the stationary behaviour of many-body systems with stochastic dynamics. The idea is that the steady state properties of the ASEP can be obtained exactly in terms of matrices obeying a quadratic algebra $[16,17]$. For a given configuration $\left(s_{1}, s_{2}, \ldots, s_{L}\right)$ the stationary probability is defined by the expectation value

$$
P(s)=\frac{\left\langle w\left|D_{s_{1}} D_{s_{2}} \cdots D_{s_{L}}\right| v\right\rangle}{Z_{L}}
$$

where $D_{s_{i}}=D_{1}$, if a site $i=1,2, \ldots, L$ is occupied and $D_{s_{i}}=D_{0}$, if a site $i$ is empty. The quantity $Z_{L}=\left\langle w\left|\left(D_{0}+D_{1}\right)^{L}\right| v\right\rangle$ is the normalization factor to the stationary probability distribution. The operators $D_{i}, i=0,1$ satisfy the quadratic (bulk) algebra (known as diffusion algebra [18])

$$
D_{1} D_{0}-q D_{0} D_{1}=x_{1} D_{0}-D_{1} x_{0}, \quad x_{0}+x_{1}=0
$$

with boundary conditions of the form

$$
\left(\beta D_{1}-\delta D_{0}\right)|v\rangle=x_{0}|v\rangle, \quad\langle w|\left(\alpha D_{0}-\gamma D_{1}\right)=-\langle w| x_{1}
$$

and $\langle w \mid v\rangle \neq 0$. The boundary conditions define the two vectors $\langle w|$ and $|v\rangle$ which enter the expectations values for the stationary weights. The open model with boundary processes depends on five parameters. These are the bulk probability rate and the four boundary rates. The partially asymmetric exclusion process (PASEP) corresponds to $0<q<1$. The limit cases $q=1$ and $q=0$ are the symmetric exclusion process (SSEP) and the totally asymmetric exclusion process (TASEP). In the case of TASEP the boundary processes depend on two boundary rates corresponding to incoming particles at the left end of the chain and outgoing particles at the right end.

Given the representations of the quadratic algebra and the boundary vectors, one can evaluate all the relevant physical quantities, such as the mean density at a site $i, i=1, \ldots, L,\left\langle s_{i}\right\rangle=$ $\frac{\left\langle w\left|\left(D_{0}+D_{1}\right)^{i-1} D_{1}\left(D_{0}+D_{1}\right)^{L-i}\right| v\right\rangle}{Z_{L}}$, correlation functions, the current $J$ through a bond between site $i$ and site $i+1$ which has a very simple form $J=\frac{Z_{L-1}}{Z_{L}}$. In most studied examples one uses infinitedimensional representations of the quadratic algebra. Finite-dimensional representations [11, 19] have been considered too and they simplify calculations. Due to a constraint on the model parameters they define an invariant subspace of the infinite matrices and give exact results only on some special curves of the phase diagram.

Exact results for the ASEP with open boundaries were obtained within the MPA through the relation of the stationary state to $q$-Hermite [20] and Al-Salam-Chihara polynomials [21] in 
the case $\gamma=\delta=0$ and to the Askey-Wilson polynomials [22] in the general case. The MPA was readily generalized to many-species models [23] and to dynamical MPA [24].

Emphasizing the dependence of the open ASEP in the stationary state on the boundary rates and the equivalence with the spin $1 / 2 X X Z$ chain we can represent [25] the boundary operators in the form

$$
\beta D_{1}-\delta D_{0}=A+x_{0} \frac{\beta-\delta}{1-q}, \quad \alpha D_{0}-\gamma D_{1}=A^{*}+x_{0} \frac{\alpha-\gamma}{1-q},
$$

where $A, A^{*}$ are linear combinations of the $U_{q}(\hat{s} u(2))$ generators in the evaluation representation

$$
\begin{aligned}
& A=-\frac{x_{1} \beta}{\sqrt{1-q}} q^{N / 2} A_{+}-\frac{x_{0} \delta}{\sqrt{1-q}} A_{-} q^{N / 2}-\frac{x_{1} \beta q^{1 / 2}+x_{0} \delta}{1-q} q^{N} \\
& A^{*}=+\frac{x_{0} \alpha}{\sqrt{1-q}} q^{-N / 2} A_{+}+\frac{x_{1} \gamma}{\sqrt{1-q}} A_{-} q^{-N / 2}+\frac{x_{0} \alpha q^{-1 / 2}+x_{1} \gamma}{1-q} q^{-N} .
\end{aligned}
$$

The operators $N, A_{ \pm}$generate the $U_{q}(s u(2))$ algebra $(0<q<1)$

$$
\left[N, A_{ \pm}\right]= \pm A_{ \pm}, \quad\left[A_{-}, A_{+}\right]=\frac{q^{N}-q^{-N}}{q^{1 / 2}-q^{-1 / 2}}
$$

with a central element $Q=A_{+} A_{-}-\frac{q^{N-1 / 2}-q^{-N+1 / 2}}{\left(q^{1 / 2}-q^{-1 / 2}\right)^{2}}$. The pair of operators $A, A^{*}$ satisfy the relations of the boundary Askey-Wilson algebra

$$
\left[\left[A, A^{*}\right]_{q}, A\right]_{q}=-\rho A^{*}-\omega A-\eta, \quad\left[A^{*},\left[A, A^{*}\right]_{q}\right]_{q}=-\rho^{*} A-\omega A^{*}-\eta^{*}
$$

with structure constants

$$
\begin{aligned}
& \rho=x_{0}^{2} \beta \delta q^{-1}\left(q^{1 / 2}+q^{-1 / 2}\right)^{2}, \quad \rho^{*}=x_{0}^{2} \alpha \gamma q^{-1}\left(q^{1 / 2}+q^{-1 / 2}\right)^{2}, \\
& -\omega=x_{0}^{2}(\beta-\delta)(\gamma-\alpha)-x_{0}^{2}(\beta \gamma+\alpha \delta)\left(q^{1 / 2}-q^{-1 / 2}\right) Q \\
& \eta=q^{1 / 2}\left(q^{1 / 2}+q^{-1 / 2}\right) x_{0}^{3}\left(\beta \delta(\gamma-\alpha) Q+\frac{(\beta-\delta)(\beta \gamma+\alpha \delta)}{q^{1 / 2}-q^{-1 / 2}}\right), \\
& \eta^{*}=q^{1 / 2}\left(q^{1 / 2}+q^{-1 / 2}\right) x_{0}^{3}\left(\alpha \gamma(\beta-\delta) Q+\frac{(\alpha-\gamma)(\alpha \delta+\beta \gamma)}{q^{1 / 2}-q^{-1 / 2}}\right) .
\end{aligned}
$$

Throughout the text $[X, Y]_{q}=q^{1 / 2} X Y-q^{-1 / 2} Y X$. We note the relative difference of the factor $q^{1 / 2}$ in $\rho\left(q^{-1 / 2}\right.$ in $\left.\rho^{*}\right)$ compared to [25] which is simply due to a rescaling of the generators $A, A^{*}$. It is important to stress (see next section) that the Askey-Wilson algebra is defined up to an affine transformation of rescaling the generators by scalars $t, t^{*}$. This property can be used to even hide the parameters $\beta \delta$ in $\rho\left(\alpha \gamma\right.$ in $\left.\rho^{*}\right)$. It is known $[26,27]$ that the infinitedimensional irreducible modules of the AW algebra are the AW polynomials depending on four parameters $a, b, c, d$. In the basic representation one $A^{*}$ is represented by a diagonal and $A-$ by a tridiagonal matrix. The sign of $\rho^{*}$ (respectively of $\rho$ in the dual representation) is essential [27] for the spectrum of the diagonal operator. Since for a representation of the AW algebra we use the $U_{q}(s u(2))$ algebra, which is the limit case of a $(u,-u), u<0$ algebra, the spectrum of the diagonal operator is of the form sinh (see [25] for the details). The boundary conditions of the ASEP uniquely relate the four parameters of the AW polynomials to the four boundary rates $a=\kappa_{+}^{*}(\alpha, \gamma), b=\kappa_{+}(\beta, \delta), c=\kappa_{-}^{*}(\alpha, \gamma), d=\kappa_{-}(\beta, \delta)$, where $\kappa_{ \pm}^{(*)}(\nu, \tau)$ is

$$
\kappa_{ \pm}^{(*)}(\nu, \tau)=\frac{-(\nu-\tau-(1-q)) \pm \sqrt{(\nu-\tau-(1-q))^{2}+4 \nu \tau}}{2 \nu} .
$$


If we use the $(u, u)$ algebra, the limit case of which corresponds to $q^{N}+q^{-N}$ in the nominator of equation (1), then the spectrum of the diagonal operator is of the form $\cosh$ and $\kappa_{ \pm}^{(*)}(\nu, \tau) \rightarrow$ $\kappa_{ \pm}^{(*)}(\nu,-\tau)$

The transfer matrix $D_{0}+D_{1}$ and each of the boundary operators generate isomorphic AW algebras [25]. In the tridiagonal representation the transfer matrix $D_{0}+D_{1}$ satisfies the threeterm recurrence relation of the AW polynomials, which was explored in [22] for the solution of the ASEP in the stationary state. The exact calculation of all the physical quantities, such as the current, correlation functions etc, in terms of the Askey-Wilson polynomials was achieved without any reference to the AW algebra. The exact solution in the stationary state in terms of the AW polynomials was, in our opinion, possible due to the appearance of the AW algebra as the boundary hidden symmetry of the ASEP with general boundary conditions. We have constructed an AW algebra operator valued $K$-matrix [14], a solution to the boundary Yang-Baxter equation. The relation of the boundary AW algebra to the $K$-matrix, satisfying the reflection equation, reveals deep algebraic properties of the ASEP allowing to extend the exact solvability beyond the stationary state. The boundary Yang-Baxter equation is the basic ingredient of the inverse scattering method [15] and one can use its solutions for description of the dynamics of the process. With the help of the $K(z)$-matrix one can apply the technique of the quantum inverse scattering method to construct the spectral dependent transfer matrix and then implement the underlying Bethe Ansatz method to exactly calculate some quantities of physical interest, e.g. the exact spectrum of the transition matrix $\Gamma$, or equivalently the Hamiltonian.

\section{Bulk tridiagonal algebra of the simple exclusion process}

For completeness we first recall the bulk algebra of the partially asymmetric exclusion process (PASEP).

The bulk tridiagonal algebra of PASEP: The operators $D_{0}, D_{1}$ of the partially asymmetric exclusion process and their $q$-commutator $\left[D_{0}, D_{1}\right]_{q}$ form a closed linear algebra

$$
\begin{aligned}
{\left[D_{0}, D_{1}\right]_{q}=D_{2}, } & \\
{\left[D_{1},\left[D_{0}, D_{1}\right]_{q}\right]_{q}=} & q^{-1 / 2} x_{1}\left(q^{1 / 2}-q^{-1 / 2}\right)\left\{D_{0}, D_{1}\right\} \\
& -q^{-1} x_{1}^{2} D_{0}+q^{-1} x_{0} x_{1} D_{1}-x_{0} q^{-1 / 2}\left(q^{1 / 2}-q^{-1 / 2}\right) D_{1}^{2}, \\
{\left[\left[D_{0}, D_{1}\right]_{q}, D_{0}\right]_{q}=} & -x_{0} q^{-1 / 2}\left(q^{1 / 2}-q^{-1 / 2}\right)\left\{D_{0}, D_{1}\right\} \\
& -x_{0}^{2} q^{-1} D_{1}+x_{0} x_{1} q^{-1} D_{0}-x_{1} q^{-1 / 2}\left(q^{1 / 2}-q^{-1 / 2}\right) D_{0}^{2},
\end{aligned}
$$

where $\left\{D_{0}, D_{1}\right\}=D_{0} D_{1}+D_{1} D_{0}$. The algebra can equivalently be described as a two-relation algebra for the pair $D_{0}, D_{1}$

$$
\begin{aligned}
& D_{0} D_{1}^{2}-\left(q+q^{-1}\right) D_{1} D_{0} D_{1}+D_{1}^{2} D_{0}+x_{1} q^{-1 / 2}\left(q^{1 / 2}-q^{-1 / 2}\right)\left\{D_{0}, D_{1}\right\} \\
& \quad=x_{1}^{2} q^{-1} D_{0}-x_{0} x_{1} q^{-1} D_{1}+x_{0} q^{-1 / 2}\left(q^{1 / 2}-q^{-1 / 2}\right) D_{1}^{2} \\
& D_{0}^{2} D_{1}-\left(q+q^{-1}\right) D_{0} D_{1} D_{0}+D_{1} D_{0}^{2}-x_{0} q^{-1 / 2}\left(q^{1 / 2}-q^{-1 / 2}\right)\left\{D_{0}, D_{1}\right\} \\
& \quad=x_{0}^{2} q^{-1} D_{1}-x_{0} x_{1} q^{-1} D_{0}+x_{1} q^{-1 / 2}\left(q^{1 / 2}-q^{-1 / 2}\right) D_{0}^{2} .
\end{aligned}
$$

The above relations are the well known Askey-Wilson relations

$$
\begin{aligned}
& A^{2} A^{*}-\left(q+q^{-1}\right) A A^{*} A+A^{*} A^{2}-\gamma\left(A A^{*}+A^{*} A\right)=\rho A^{*}+\gamma^{*} A^{2}+\omega A+\eta, \\
& A^{* 2} A-\left(q+q^{-1}\right) A^{*} A A^{*}+A A^{* 2}-\gamma^{*}\left(A A^{*}+A^{*} A\right)=\rho^{*} A+\gamma A^{* 2}+\omega A^{*}+\eta^{*} .
\end{aligned}
$$

The algebra (4) was first considered in the works of Zhedanov [27, 28] who showed that the Askey-Wilson polynomials give pairs of infinite-dimensional matrices satisfying the AskeyWilson (AW) relations. It is recently discussed in a more general framework of a tridiagonal 
algebra $[29,30]$, that is an associative algebra with a unit generated by a (tridiagonal) pair of operators $A, A^{*}$ and defining relations, obtained by taking the commutator with $A, A^{*}$ in the first (second) line of (4) respectively

$$
\begin{aligned}
& {\left[A, A^{2} A^{*}-\beta A A^{*} A+A^{*} A^{2}-\gamma\left(A A^{*}+A^{*} A\right)-\rho A^{*}\right]=0,} \\
& {\left[A^{*}, A^{* 2} A-\beta A^{*} A A^{*}+A A^{* 2}-\gamma^{*}\left(A A^{*}+A^{*} A\right)-\rho^{*} A\right]=0 .}
\end{aligned}
$$

In the general case a tridiagonal pair is determined by the sequence of scalars $\beta, \gamma, \gamma^{*}, \rho, \rho^{*}$. (We keep the conventional notations, used in the literature, for the scalars of a tridiagonal pair; $\beta$ and $\gamma$ should not be confused with the ASEP boundary rates.) Tridiagonal pairs have been classified according to the dependence on the scalars [29]. The example which is important for the present study are the Dolan-Grady relations [31] with $\beta=2, \gamma=\gamma^{*}=0, \rho=k^{2}, \rho^{*}=k^{* 2}$

$$
\left[A,\left[A,\left[A, A^{*}\right]\right]\right]=k^{2}\left[A, A^{*}\right], \quad\left[A^{*},\left[A^{*},\left[A^{*}, A\right]\right]\right]=k^{* 2}\left[A^{*}, A\right] .
$$

Tridiagonal pairs are determined up to an affine transformation

$$
A \rightarrow t A+c, \quad A^{*} \rightarrow t^{*} A^{*}+c^{*},
$$

where $t, t^{*}, c, c^{*}$ are some scalars. The affine transformation can be used to bring a tridiagonal pair in a reduced form with $\gamma=\gamma^{*}=0$.

As seen from the Askey-Wilson relations (4) for the matrices $D_{0}$ and $D_{1}$ we have explicitly

$$
\begin{aligned}
& \rho=x_{1}^{2} q^{-1}, \quad \rho^{*}=x_{0}^{2} q^{-1}, \quad \omega=-x_{0} x_{1} q^{-1}, \\
& \gamma=-x_{1} q^{-1 / 2}\left(q^{1 / 2}-q^{-1 / 2}\right), \quad \gamma^{*}=x_{0} q^{-1 / 2}\left(q^{1 / 2}-q^{-1 / 2}\right)
\end{aligned}
$$

and $\eta=\eta^{*}=0$. Besides $\gamma=\gamma^{*}, \rho=\rho^{*}$ due to $x_{0}+x_{1}=0$. We can now rescale the operators $D_{0}, D_{1}$ to set $\gamma=\gamma^{*}=0$. This is achieved with the help of the transformations

$$
D_{0} \rightarrow D_{0}+\frac{x_{0} q^{-1 / 2}}{q^{1 / 2}-q^{-1 / 2}}, \quad D_{1} \rightarrow D_{1}-\frac{x_{1} q^{-1 / 2}}{q^{1 / 2}-q^{-1 / 2}}
$$

However the shift of the generators amounts to a tridiagonal pair with sequence of scalars $\beta=-\left(q+q^{-1}\right), \gamma=\gamma^{*}=0, \rho=\rho^{*}=0$. Thus the operators of the ASEP matrix product ansatz obey the relations of a tridiagonal algebra

$$
\begin{aligned}
& {\left[D_{1}, D_{0} D_{1}^{2}-\left(q+q^{-1}\right) D_{1} D_{0} D_{1}+D_{1}^{2} D_{0}\right]=0,} \\
& {\left[D_{0}, D_{1} D_{0}^{2}-\left(q+q^{-1}\right) D_{0} D_{1} D_{0}+D_{0}^{2} D_{1}\right]=0,}
\end{aligned}
$$

which is a special case of the tridiagonal relations of the ASEP boundary operators with $\beta=$ $q+q^{-1}$ and $\gamma=\gamma^{*}=\rho=\rho^{*}=0$. These relations are the $q$-Serre relations for the level zero $U_{q}(\hat{s} u(2))$ adjoint representation. As already mentioned we have defined the Askey-Wilson algebra with two generators through a homomorphism to the quantized affine $U_{q}(\hat{s u}(2))$ [14]. It is important to understand the role of the parameters $x_{0}, x_{1}$ in the quadratic algebra of the open ASEP from the point of view of this homomorphism. These parameters can be interpreted as the weights associated to the Cartan generators $H_{i}, i=0,1$ of the $U_{q}(\hat{s} u(2))$ finite-dimensional module with weight space $W_{\nu}=\left(\nu \in W \mid q^{H_{i}} \nu=q^{x_{i}} \nu\right)$. The $U_{q}(\hat{s u}(2))$ module $W$ has level $k$ if the central element $q^{H_{0}+H_{1}}$ acts on it as the scalar $q^{k}$ [33]. Hence, it follows from the bulk tridiagonal algebra (5) that the matrices $D_{0}, D_{1}$ of the quadratic bulk algebra of the open ASEP with $x_{0}+x_{1}$ correspond to the two-dimensional level $0 U_{q}(\hat{s u}(2))$ adjoint module.

The bulk Askey-Wilson algebra of the symmetric exclusion process follows immediately as the limit $q \rightarrow 1$ of the bulk tridiagonal algebra of the asymmetric exclusion process. Hence we have: 
Proposition 1. The operators $D_{0}, D_{1}$ of the symmetric simple exclusion process and their commutator $\left[D_{0}, D_{1}\right]$ form a closed linear algebra

$$
\begin{aligned}
& {\left[D_{0}, D_{1}\right]=D_{2}, \quad\left[D_{1},\left[D_{0}, D_{1}\right]\right]=x_{1}\left\{D_{0}, D_{1}\right\}-x_{1}^{2} D_{0}+x_{0} x_{1} D_{1}-x_{0} D_{1}^{2},} \\
& {\left[\left[D_{0}, D_{1}\right], D_{0}\right]=-x_{0}\left\{D_{0}, D_{1}\right\}-x_{0}^{2} D_{1}+x_{0} x_{1} D_{0}-x_{1} D_{0}^{2} .}
\end{aligned}
$$

The proposition can be independently verified by directly using the explicit form of the MPA quadratic relation for the case of symmetric diffusion. The algebra can equivalently be described as a two-relation algebra for the pair $D_{0}, D_{1}$

$$
\begin{aligned}
& D_{0} D_{1}^{2}-2 D_{1} D_{0} D_{1}+D_{1}^{2} D_{0}+x_{1}\left\{D_{0}, D_{1}\right\}=x_{1}^{2} D_{0}-x_{0} x_{1} D_{1}+x_{0} D_{1}^{2}, \\
& D_{0}^{2} D_{1}-2 D_{0} D_{1} D_{0}+D_{1} D_{0}^{2}-x_{0}\left\{D_{0}, D_{1}\right\}=x_{0}^{2} D_{1}-x_{0} x_{1} D_{0}+x_{1} D_{0}^{2} .
\end{aligned}
$$

As seen from the explicit form of the algebra, the matrices $D_{0}, D_{1}$ of the symmetric exclusion process satisfy the Askey-Wilson relations with the sequence of scalars

$$
\beta=2, \quad \rho=x_{1}^{2}, \quad \rho^{*}=x_{0}^{2}, \quad \omega=-x_{0} x_{1}, \quad \gamma=-x_{1}, \quad \gamma^{*}=-x_{0} .
$$

We can transform the generators $D_{0}, D_{1}$ by the affine shifts

$$
D_{0} \rightarrow D_{0}+x_{0}, \quad D_{1} \rightarrow D_{1}-x_{1} .
$$

By a straightforward calculation we again observe the same property as in the case of the partially asymmetric process. Namely, the shifts of the generators amount to a tridiagonal pair with the sequence of scalars $\gamma=\gamma^{*}=0, \rho=\rho^{*}=0$. Thus the operators of the symmetric matrix product ansatz obey the relations of a tridiagonal algebra

$$
\left[D_{1}, D_{0} D_{1}^{2}-2 D_{1} D_{0} D_{1}+D_{1}^{2} D_{0}\right]=0, \quad\left[D_{0}, D_{1} D_{0}^{2}-2 D_{0} D_{1} D_{0}+D_{0}^{2} D_{1}\right]=0,
$$

which are the Dolan-Grady relations with $k=k^{*}=0$. In the next section we show that the bulk tridiagonal relations are the special case of the tridiagonal relations obeyed by the boundary operators of the symmetric process.

\section{Boundary algebra of the symmetric exclusion process}

We consider the symmetric simple exclusion process (SSEP) with most general boundary conditions of incoming and outgoing particle at both ends of the chain. Within the matrix product ansatz the quadratic bulk algebra of the SSEP is the $q=1$ limit of the deformed quadratic algebra of the ASEP. In the previous section we derived the bulk tridiagonal algebra of the symmetric process and it turned to be the $q=1$ limit of the bulk tridiagonal algebra of the asymmetric process.

We are going to obtain the boundary algebra of the symmetric process as the $q=1$ limit of the tridiagonal boundary algebra of the asymmetric simple exclusion process. We start with the observation about the natural homomorphism of the tridiagonal algebra (TA) generated by the pair $A, A^{*}$ and the Askey-Wilson algebra (AW) of the ASEP defined by equation (2), namely $T A \rightarrow A W$. As already mentioned, this is readily verified by taking the commutator with $A$, respectively $A^{*}$ of the first line, respectively the second line of equation (2), which gives

$$
\left.\left[A,\left[A,\left[A, A^{*}\right]_{q},\right]_{q^{-1}}\right]\right]=\rho\left[A, A^{*}\right], \quad\left[A^{*},\left[A^{*}\left[A^{*}, A\right]_{q}\right]_{q^{-1}}\right]=\rho^{*}\left[A^{*}, A\right]
$$

with $\rho, \rho^{*}$ depending on the five parameters of the ASEP, as given by (3). The operators $A, A^{*}$ were introduced as the shifted boundary operators of the ASEP. The limit $q=1$ of the tridiagonal algebra (6) provides a way to determine the boundary algebra of the SSEP. 
The boundary operators of the SSEP can be represented in the form (i.e. linear combinations in terms of the level zero affine $s u(2)$ generators)

$$
\begin{aligned}
& \beta D_{1}-\delta D_{0}=-x_{1} \beta A_{+}-x_{0} \delta A_{-}-\left(x_{1} \beta+x_{0} \delta\right) N-x_{1} \beta-x_{0} \delta \\
& \alpha D_{0}-\gamma D_{1}=x_{0} \alpha A_{+}+x_{1} \gamma A_{-}+\left(x_{0} \alpha+x_{1} \gamma\right) N+x_{0} \alpha+x_{1} \gamma
\end{aligned}
$$

where $A_{ \pm}, N$ are corresponding operators in the limit $q \rightarrow 1$ of equation (1). We separate the shift parts from the boundary operators. Denoting the corresponding rest operator parts by $A$ and $A^{*}$ we write the left and right boundary operators in the form

$$
\beta D_{1}-\delta D_{0}=A-x_{1} \beta-x_{0} \delta, \quad \alpha D_{0}-\gamma D_{1}=A^{*}+x_{0} \alpha+x_{1} \gamma
$$

Then we have the following

Proposition 2. The operators $A$ and $A^{*}$ defined by the corresponding shifts of the boundary operators of the open symmetric exclusion process

$$
A=\beta D_{1}-\delta D_{0}+\left(x_{1} \beta+x_{0} \delta\right), \quad A^{*}=\alpha D_{0}-\gamma D_{1}-\left(x_{0} \alpha+x_{1} \gamma\right)
$$

and their commutator

$$
\left[A, A^{*}\right]=A A^{*}-A^{*} A
$$

form a closed linear algebra, the boundary tridiagonal algebra of the SSEP

$$
\left[A,\left[A,\left[A, A^{*}\right]\right]\right]=\rho\left[A, A^{*}\right], \quad\left[A^{*},\left[A^{*},\left[A^{*}, A\right]\right]\right]=\rho^{*}\left[A^{*}, A\right],
$$

where the structure constants are given by

$$
-\rho=x_{0} x_{1} \beta \delta, \quad-\rho^{*}=x_{0} x_{1} \alpha \gamma
$$

This proposition is straightforward to verify by taking the $q \rightarrow 1$ limit in the chain of homomorphisms $T D \rightarrow A W \rightarrow U_{q}(\hat{s u}(2))$ or independently by directly using equation (7).

As readily seen from the definition (8) the (shifted) boundary operators of the symmetric exclusion process obeying the algebra (9) form a tridiagonal pair with $\beta=2, \gamma=\gamma^{*}=0$, and $\rho, \rho^{*}$ given by (10). The tridiagonal boundary algebra of the symmetric process is the limit $q=1$ of the deformed boundary algebra of the ASEP as the irreducible modules of the algebra in the symmetric model, i.e. the Wilson polynomials, are the $q=1$ limit of the Askey-Wilson polynomials. The important properties of the deformed Askey-Wilson algebra remain valid in the proper limit $q \rightarrow 1$. Stated more precisely which will correspond to the historical development of these algebras, for generic $q$ the deformed Askey-Wilson algebra is the q-generalization of the Onsager algebra in the form of the Dolan-Grady relations. Its irreducible modules, i.e. the Askey-Wilson polynomials are $q$-counterpart of the Wilson polynomials. For applications, however one can even use the limit cases of the Wilson polynomials. These are the continuous Hahn and dual Hahn polynomials (see [34] and [35] for details on these polynomials). In both cases there exist limiting procedures to further obtain the Meixner-Pollaczek polynomials from which the Laguerre polynomials can be obtained. Let $P_{n}^{\mu}(x, \phi)$ denote the $n$-th Meixner-Pollaczek

$$
P_{n}^{\mu}(x, \phi)=\frac{(2 \mu)_{n}}{n !} e^{i n \phi}{ }_{2} F_{1}\left(\begin{array}{c}
-n, \mu+i x \\
2 \mu
\end{array} \mid 1-e^{-2 i \phi}\right) .
$$

The Laguerre polynomials can be obtained by the substitution $\mu=1 / 2(\lambda+1), x \rightarrow-1 / 2 \phi^{-1}(x)$ and letting $\phi \rightarrow 0$

$$
\lim _{\phi \rightarrow 0} P_{n}^{1 / 2 \mu+1 / 2}\left(-\frac{x}{2 \phi} ; \phi\right)=L_{n}^{(\lambda)}(x)
$$


By definition the Laguerre polynomials have the form

$$
L_{n}^{(\lambda)}(x)=\frac{(\lambda+1)_{n}}{n !}{ }_{1} F_{1}\left(\begin{array}{c}
-n \\
\lambda+1
\end{array} \mid x\right)
$$

with orthogonality condition

$$
\int_{0}^{\infty} e^{-x} x^{\lambda} L_{m}^{\lambda} L_{n}^{\lambda} d x=\frac{\Gamma(n+\lambda+1)}{n !} \delta_{m n}, \quad \lambda>-1
$$

and recurrence relation

$$
(n+1) L_{n+1}^{(\lambda)}(x)-(2 n+\lambda+1-x) L_{n}^{(\lambda)}(x)+(n+\lambda) L_{n-1}^{(\lambda)}(x)=0,
$$

where $L_{-1}^{(\lambda)}(x)=0$ and $L_{0}^{(\lambda)}(x)=1$. One can identify

$$
\lambda=\frac{\alpha+\beta+\gamma+\delta}{(\alpha+\gamma)(\beta+\delta)}-1
$$

Denoting

$$
l_{n}(x)=(-1)^{n}\left(\frac{n ! \Gamma(\lambda+1)}{\Gamma(\lambda+n+1)}\right)^{1 / 2} L_{n}^{(\lambda)}(x)
$$

we rewrite the orthogonality condition in the form

$$
1=\frac{1}{\Gamma(\lambda+1)} \int_{0}^{\infty} e^{-x} x^{\lambda}|l(x)\rangle\langle l(x)| d x .
$$

The vectors $|l(x)\rangle=\left(l_{0}(x), l_{1}(0), \ldots\right)^{t}$ and $\langle l(x)|=\left(l_{0}(x), l_{1}(0), \ldots\right)$ form the basis for the tridiagonal and the diagonal representation of the generators (and for the dual one). As it was proved to be the case for the ASEP [25], each boundary operator of the symmetric process, together with the transfer matrix operator $D_{0}+D_{1}$, forms an isomorphic AW algebra whose tridiagonal representation follows from the three-term recurrence relation, consistent with the orthogonality condition

$$
\left(D_{0}+D_{1}\right)|l(x)\rangle=x|l(x)\rangle, \quad\langle l(x)|\left(D_{0}+D_{1}\right)=\langle l(x)| x .
$$

These properties can be used to exactly calculate the physical quantities [22] in the stationary state. The results [2] for the partition function $Z_{l}$ and the current are reconstructed

$$
Z_{L}=\frac{\Gamma(\lambda+L+1)}{\Gamma(\lambda+1)}, \quad J=\frac{1}{\lambda+L}
$$

The one point function [2]

$$
\left\langle\tau_{i}\right\rangle=\frac{\alpha}{\alpha+\gamma}-\frac{1}{\lambda+L} \frac{\alpha \beta-\gamma \delta}{(\alpha+\gamma)(\beta+\delta)}\left(\frac{1}{\alpha+\gamma}+i-1\right), \quad i=1,2, \ldots, L
$$

shows that the particle density has a linear profile. Without reference to the AW algebra the eigenvalue equation (11) was used in [22] to calculate the physical quantities of the symmetric exclusion process, as known from the matrix product approach. In our opinion it is the boundary tridiagonal algebra of the symmetric exclusion process that allows for the exact solvability of the symmetric process in the stationary state and leads to a generalization of the matrix product method. 


\section{Nonlocal conserved charges of the symmetric exclusion process}

In the previous section we have shown that the boundary symmetry of the symmetric exclusion process is the tridiagonal algebra (9) with the sequence of scalars $\beta=2, \rho, \rho^{*}$. This algebra is the $q=1$ limit of the tridiagonal algebra mapped through the natural homomorphism to the Zhedanov algebra $A W(3)$ defined for $0<q<1$. The exact calculation of the physical quantities in the case of the symmetric exclusion process has been obtained in terms of the Laguerre polynomials implementing [22] the three-term recurrence relation to define the tridiagonal representation of the transfer matrix $D_{0}+D_{1}$. As we pointed out, the ultimate relation of the exact solution in the stationary state to the Laguerre polynomials was possible due to the $q=1$ boundary hidden symmetry of the SSEP with general boundary conditions.

The defining relations (9) of the $q=1$ tridiagonal boundary algebra can (with $\rho, \rho^{*}$ expressed in terms of the boundary rates according to (10)) are the well-known Dolan-Grady relations for the shifted boundary operators. The importance of the Dolan-Grady relations is that given a self-dual Hamiltonian

$$
H=f A+f^{*} A^{*},
$$

where $f, f^{*}$ are some coupling constants, and $A, A^{*}$ satisfy the relations (9), then one can construct (an infinite set of) conserved commuting charges

$$
Q_{2 n}=f\left(R_{2 n}-\tilde{R}_{2 n-2}\right)+f^{*}\left(\tilde{R}_{2 n}-R_{2 n-2}\right)
$$

in terms of the quantities

$$
R_{2 n}=-\frac{2}{\rho}\left[A\left[A^{*}, R_{2 n-2}\right]\right]-\tilde{R}_{2 n-2}
$$

with $R_{0} \equiv A, Q_{0} \equiv H$. In the case of the symmetric exclusion process from the boundary conditions we have

$$
\left\langle w\left|A-\left(x_{1} \beta+x_{0} \delta\right)\right| v\right\rangle=x_{0}\langle w \mid v\rangle, \quad\left\langle w\left|A^{*}+\left(x_{0} \alpha+x_{1} \gamma\right)\right| v\right\rangle=-x_{1}\langle w| v .
$$

Hence

$$
\left\langle w\left|x_{0}\left(A^{*}+\left(x_{0} \alpha+x_{1} \gamma\right)\right)+x_{1}\left(A-\left(x_{0} \beta+x_{1} \delta\right)\right)\right| v\right\rangle=0
$$

and we can interpret the quantity

$$
x_{0}\left(A^{*}+\left(x_{0} \alpha+x_{1} \gamma\right)\right)+x_{1}\left(A-\left(x_{0} \beta+x_{1} \delta\right)\right)
$$

as the Hamiltonian of the symmetric simple exclusion process in the auxiliary space. As we know the generators of the tridiagonal algebra are determined up to shift transformations. In view of this property it seems more convenient to consider the shifted Hamiltonian

$$
H_{s}=x_{0} A^{*}+x_{1} A,
$$

which is self-dual, if we define $x_{1}^{*}=x_{0}$. Then we can straightforward apply the prescription of Dolan and Grady to obtain the conserved nonlocal charges. Taking into account the shifts we find the result

$$
R_{0}=A-x_{1} \beta-x_{0} \delta, \quad \tilde{R}_{0}=A^{*}+x_{0} \alpha+x_{1} \gamma
$$




$$
R_{2}=-\frac{2}{\rho}\left[A-x_{1} \beta-x_{0} \delta,\left[A^{*}+x_{0} \alpha+x_{1} \gamma, A-x_{1} \beta-x_{0} \delta\right]\right]-A^{*}+x_{0} \alpha+x_{1} \gamma
$$

and so on, according to formula (12). The expressions $A-x_{1} \beta-x_{0} \delta$ and $A^{*}+x_{0} \alpha+x_{1} \gamma$ are the right and left boundary operators

$$
B^{R}=\beta D_{1}-\delta D_{0}, \quad B^{L}=\alpha D_{1}-\gamma D_{0}
$$

respectively which acquire a very important physical meaning. The boundary operators satisfying

$$
\begin{aligned}
& {\left[B^{R},\left[B^{R},\left[B^{R}, B^{L}\right]\right]\right]=-x_{0} x_{1} \beta \delta\left[B^{R}, B^{L}\right],} \\
& {\left[B^{L},\left[B^{L},\left[B^{L}, B^{R}\right]\right]\right]=-x_{0} x_{1} \alpha \gamma\left[B^{L}, B^{R}\right]}
\end{aligned}
$$

are the nonlocal conserved charges of the symmetric exclusion process with the help of which the (infinite) set of conserved quantities of the process are constructed. By properly rescaling the operators one achieves equal coefficient factors on the RHS of equations (13). We note that quantum integrals of motion for the $X X X$ Heisenberg infinite chain (known to be related to the symmetric exclusion process) were first obtained in [32]. For the open symmetric exclusion process the existence of nonlocal conserved quantities will result in the exact solvability of the system beyond the stationary state due to the boundary hidden tridiagonal symmetry of the symmetric exclusion process.

\section{Askey-Wilson algebra of the totally asymmetric exclusion process}

The tridiagonal and the AW algebra of the totally asymmetric exclusion process cannot be obtained directly as the limit $q=0$ of the partially asymmetric process. The procedure is more involved. We derive this algebra from the quadratic algebra of the totally asymmetric process.

We start with the quadratic algebra

$$
D_{1} D_{0}=D_{1}+D_{0}
$$

From this algebra the following relations follow

$$
D_{1} D_{0} D_{1}=D_{1}^{2}+D_{0} D_{1}, \quad D_{0} D_{1} D_{0}=D_{0} D_{1}+D_{0}^{2}
$$

and

$$
D_{1}^{2} D_{0}=D_{1}^{2}+D_{1}+D_{0}, \quad D_{1} D_{0}^{2}=D_{1}+D_{0}+D_{0}^{2},
$$

which can alternatively be written as

$$
D_{1} D_{0} D_{1}-D_{1}^{2} D_{0}=\left[D_{0}, D_{1}\right], \quad D_{0} D_{1} D_{0}-D_{1} D_{0}^{2}=\left[D_{0}, D_{1}\right] .
$$

The LHS of (17) are respectively

$$
\left[D_{1} D_{0}, D_{1}\right], \quad\left[D_{0}, D_{1} D_{0}\right]
$$

Hence we have

$$
D_{1}\left[D_{0}, D_{1}\right]=\left[D_{0}, D_{1}\right], \quad\left[D_{0}, D_{1}\right] D_{0}=\left[D_{0}, D_{1}\right] .
$$


Examples of matrices obeying the above relations are given by the equation (6) and (36), (38) in [16]. We can shift the operators $D_{0}, D_{1}$ by 1 (or respectively by the constants $c_{0}=a, c_{1}=b$ )

$$
D_{0} \rightarrow D_{0}+1, \quad D_{1} \rightarrow D_{1}+1 .
$$

Then we can write subsequently $D_{1} D_{0}=Z$, where either $Z=1$ or $Z=a b$. (In the case of the totally asymmetric exclusion process within the matrix product approach $a b=\alpha \beta$, where $\alpha$ and $\beta$ are the probability rates for the particles to be added and removed at both sides of the linear chain.) Hence

$$
\begin{aligned}
& D_{1} D_{0} D_{1}=Z D_{1}, \quad D_{0} D_{1} D_{0}=Z D_{0}, \\
& D_{1}^{2} D_{0}=Z D_{1}, \quad D_{1} D_{0}^{2}=Z D_{0} .
\end{aligned}
$$

Relations (17) for the shifted generators become

$$
D_{1} D_{0} D_{1}-D_{1}^{2} D_{0}=0, \quad D_{0} D_{1} D_{0}-D_{1} D_{0}^{2}=0
$$

and consequently

$$
D_{1}\left[D_{0}, D_{1}\right]=0, \quad\left[D_{0}, D_{1}\right] D_{0}=0 .
$$

We need to emphasize that multiplying in equations (22), the first equation by $D_{1}$ from the right and the second equation by $D_{0}$ from the left, we obtain

$$
D_{1} D_{0} D_{1}^{2}-D_{1}^{2} D_{0} D_{1}=0, \quad D_{0}^{2} D_{1} D_{0}-D_{0} D_{1} D_{0}^{2}=0,
$$

which define the $q=0$ limit of the $q$-Serre relations of $U_{q}(\hat{s l}(2))$, i.e. the $q=0$ limit of the level zero $U_{q}(\hat{s l}(2))$ adjoint representation. This is consistent with the definition of the Askey-Wilson algebra such, that it yields a deformation of the level zero $U_{q}(\hat{s l}(2)) q$-Serre relations (see [14] for details). However, we have now the additional relation

$$
\left(\left[D_{0}, D_{1}\right]\right)^{2}=Z\left[D_{0}, D_{1}\right] .
$$

It is important to emphasize that the matrices obeying (14)-(19) and (20)-(23) are upper bidiagonal and lower bidiagonal. It is known that the basic representation of the AW algebra can be equivalently considered as a representation on the space of $c$-number sequences where one of the generators acts as a tridiagonal operator, while the other generator acts as a diagonal operator. In our case the analogues of the diagonal and tridiagonal matrices in the $q=0$ limit of the basic representation are given by $\left[D_{0}, D_{1}\right]$ and $D_{1}+D_{0}$ respectively.

Proposition 3. The $q=0 A W$ algebra, as following from the quadratic algebra (14), depending on only two constants $a, b$ (i.e. $e_{1}=a+b, e_{2}=a b$ ) in the basic representation, is defined by

$$
\begin{aligned}
& D_{1} D_{0}=e_{2}, \quad D_{1}\left[D_{0}, D_{1}\right]=0, \quad\left[D_{0}, D_{1}\right] D_{0}=0, \\
& {\left[D_{0}, D_{1}\right]\left(D_{0}+D_{1}\right)\left[D_{0}, D_{1}\right]=0, \quad\left(\left[D_{0}, D_{1}\right]\right)^{2}=e_{2}\left[D_{0}, D_{1}\right] .}
\end{aligned}
$$

The matrix $D_{0}+D_{1}$, denoted hereafter $D$, plays the role of the tridiagonal operator, and $\left[D_{0}, D_{1}\right]$, denoted hereafter $D^{*}$, - the role of the diagonal operator, in the basic representation.

Thus we have

$$
\begin{aligned}
& {\left[D_{0}, D_{1}\right]\left(D_{0}+D_{1}\right)^{2}\left[D_{0}, D_{1}\right]=e_{2}^{2}\left[D_{0}, D_{1}\right],} \\
& {\left[D_{0}, D_{1}\right]\left(D_{0}+D_{1}\right)\left[D_{0}, D_{1}\right]=0, \quad\left(\left[D_{0}, D_{1}\right]\right)^{2}=e_{2}\left[D_{0}, D_{1}\right] .}
\end{aligned}
$$

We can now shift the matrix $\left(D_{0}+D_{1}\right) \equiv D \rightarrow D+a+b$ to obtain a tridiagonal matrix with entries on the main diagonal too. 
Definition 1. The $q=0$ limit of the AW algebra depending on only two constants $a, b$, where $e_{1}=a+b, e_{2}=a b$, is generated by a tridiagonal operator $D$ and a diagonal operator $D^{*}$ with defining relations in the basic representation

$$
D^{*} D D^{*}=e_{1} e_{2} D^{*}, \quad\left(D^{*}\right)^{2}=e_{2} D^{*} .
$$

One can alternatively consider defining relations for $q=0$ limit of the AW algebra in a representation associated with the $q=0$ limit of the level zero adjoint $U_{q}(\hat{s l}(2))$ (equation (24)).

Definition 2. The $q=0$ limit of the AW algebra, generated by upper diagonal and lower diagonal matrices $D_{1}$ and $D_{0}$ and depending on only two constants $a, b$, is defined by

$$
D_{1} D_{0} D_{1}=a b D_{1}, \quad D_{0} D_{1} D_{0}=a b D_{0} .
$$

The boundary tridiagonal AW algebra of the totally asymmetric exclusion process corresponds to the values $a=\alpha, b=\beta$ while the bulk AW algebra is obtained for $a=b=1$.

We note that the two representation dependent definitions of the algebras considered above have in common the first relation in formula (25) and both relations in (26), which can be unified as

$$
A B A=\tilde{c}(a, b) A,
$$

where $A, B$ are the generators of these algebras and $\tilde{c}(a, b)$ is a constant depending on the parameters $a, b$. We can multiply equation (27) by $B$ subsequently from the left and from the right to obtain

$$
A B A B-B A B A=0
$$

if $\tilde{c}(a, b)=0$ or

$$
A B A B-B A B A=\tilde{c}(a, b)(A B-B A)
$$

if $\tilde{c}(a, b) \neq 0$. We can consider equation (29) as defining an alternative $q=0$ limit algebra, obtained through the natural homomorphism to the $q=0$ limit Askey-Wilson algebra, with structure constant $\tilde{c}(a, b)$. These relations can be very useful for applications. Namely, with the additional condition $A^{2}=A$, which is a Hecke type relation equations (28) and (29) have the form of a reflection equation and a modified boundary Yang-Mills equation, respectively. The interpretation of equations (28) and (29) as reflection equations should be associated with the proper $R$-matrix operator depending on a parameter $t \neq q$.

Thus for the totally asymmetric exclusion process we obtain the bulk algebra

$$
D_{1} D_{0} D_{1} D_{0}-D_{0} D_{1} D_{0} D_{1}=D_{1} D_{0}-D_{0} D_{1}
$$

and the boundary algebra generated by the right $D^{R}$ and left $D^{*} L$ boundary operators

$$
D^{R}=\beta D_{1}, \quad D^{L}=\alpha D_{0}
$$

subject to the relations

$$
D^{R} D^{L} D^{R} D^{L}-D^{L} D^{R} D^{L} D^{R}=\alpha \beta\left(D^{R} D^{L}-D^{L} D^{R}\right) .
$$

It is worth studying the connection of the exact solvability of the totally asymmetric exclusion process in the stationary state to the integrability properties based on the boundary Yang-Baxter equation. The consequences of equation (30) will be to naturally extend the exact solvability beyond the stationary state. In [36] Bethe ansatz equations were derived and the exact spectrum of the transfer matrix of the totally asymmetric exclusion process was analyzed. In our opinion the boundary AW algebra is the hidden symmetry behind the Bethe ansatz solvability of the totally asymmetric exclusion process. 


\section{Conclusion}

We have studied the symmetry properties of the simple exclusion process. Our consideration extends the previously obtained results about the AW algebra (known as the $A W(3, q)$ ) Zhedanov algebra) to be the boundary hidden symmetry of the asymmetric exclusion process. We have shown that the boundary symmetry algebra of the symmetric simple exclusion process is the $q=1$ limit of the boundary tridiagonal algebra of the partially asymmetric exclusion process. It is this algebra, to be denoted $T A(q=1)$, that allows for the exact solvability of the symmetric model in the stationary state. The defining relations of the algebra $T A(q=1)$ are the known Dolan-Grady relations. The consequence of this fact is that one can define conserved nonlocal charges of the symmetric process, which allows for extending the exact solvability beyond the stationary state. We have also derived the bulk and boundary AW algebra of the totally asymmetric exclusion process, which can be viewed as a particular case of the algebra $A W(3, q=0)$. The defining relation of the boundary algebra of the totally asymmetric process leads to a boundary Yang-Baxter equation which might be interpreted again as the deep algebraic reason behind the exact solvability.

\section{Acknowledgments}

A CEI grant for participation in the Seventh International Conference "Symmetry in Nonlinear Mathematical Physics" is gratefully acknowledged. The author would like to thank the organizers for the invitation to participate the conference Symmetry-2007 and for the warm atmosphere during the stay in Kyiv.

\section{References}

[1] Privman V. (Editor), Nonequilibrium statistical mechanics in one dimension, Cambridge University Press, Cambridge, 1997.

[2] Schütz G.M., Exactly solvable models for many-body systems far from equilibrium, Phase Transitions and Critical Phenomena, Vol. 19, Academic Press, San Diego, CA, 2001.

[3] Chaichian M., Kulish P.P., Spin Hamiltonians, quantum groups and reaction-diffusion processes, in Multiple Facets of Quantization and Supersymmetry, Editors M. Olshanetsky and A. Vainstein, World Sci. Publ., River Edge, NJ, 2002, 310-319.

[4] Chowdhury D., Santen L., Schadschneider A., Statistical physics of vehicular traffic and some related systems, Phys. Rep. 329 (2000), 199-329, cond-mat/0007053.

[5] Helbing D., Traffic and related self-driven many particle systems, Rev. Modern Phys. 73 (2001), 1067-1141, cond-mat/0012229.

[6] MacDonald J.T., Gibbs J.H., Pipkin A.C., Kinetics of biopolymerization on nucleic acid templates, Biopolymers 6 (1968), 1-25.

[7] Krug J., Spohn H., Kinetic roughening of growing surfaces, in Solids Far from Equilibrium, Editor C. Godreche, Cambridge University Press, Cambridge, 1991, 412-525.

[8] Bundschuh R., Asymmetric exclusion process and extremal statistics of random sequences, Phys. Rev. E 65 (2002), 031911, 19 pages, cond-mat/9911386.

[9] Blythe R.A., Evans M.R., Nonequilibrium steady states of matrix product form: a solver's guide, J. Phys. A: Math. Theor. 40 (2007), R333-R441, arXiv:0706.1678.

[10] Krug J., Boundary-induced phase transitions in driven diffusive systems, Phys. Rev. Lett. 67 (1991), 18821885.

[11] Essler F.H.L., Rittenberg V., Representations of the quadratic algebra and partially asymmetric diffusion with open boundaries, J. Phys. A: Math. Gen. 29 (1996), 3375-3407, cond-mat/9506131.

[12] Sandow S., Schütz G.M., On $U_{q}(S U(2))$-symmetric driven diffusion, Europhys. Lett. 26 (1994), 7-13, cond-mat/9307027. 
[13] Pasquier V., Saleur H., Common structures between finite systems and conformal field theories through quantum groups, Nuclear Phys. B 330 (1990), 523-556.

[14] Aneva B., Chaichian M., Kulish P.P., From quantum affine symmetry to boundary Askey-Wilson algebra and reflection equation, J. Phys. A: Math. Theor. 41 (2008), 135201, 18 pages, arXiv:0804.1623.

[15] Kulish P.P., Sklyanin E.K., Quantum spectral transform method. Recent developments, Lecture Notes in Phys., Vol. 151, Springer, Berlin - New York, 1982, 61-119.

[16] Derrida B., Evans M.R., Hakim V., Pasquier V., Exact solution of a 1D asymmetric exclusion model using a matrix formulation, J. Phys. A: Math. Gen. 26 (1993), 1493-1517.

[17] Derrida B., An exactly soluble non-equilibrium system: the asymmetric simple exclusion process, Phys. Rep. 301 (1998), 65-83.

[18] Isaev A., Pyatov P., Rittenberg V., Diffusion algebras, J. Phys. A: Math. Gen. 34 (2001), 5815-5834, cond-mat/0103603.

[19] Mallick K., Sandow S., Finite-dimensional representations of the quadratic algebra: applications to the exclusion process, J. Phys. A: Math. Gen. 30 (1997), 4513-4526, cond-mat/9705152.

[20] Blythe R.A., Evans M.R., Colaiori F., Essler F.H.L., Exact solution of a partially asymmetric exclusion model using a deformed oscillator algebra, J. Phys. A: Math. Gen. 33 (2000), 2313-2332, cond-mat/9910242.

[21] Sasamoto T., One-dimensional partially asymmetric simple exclusion process with open boundaries: orthogonal polynomials approach, J. Phys. A: Math. Gen. 32 (1999), 7109-7131, cond-mat/9910483.

[22] Uchiyama M., Sasamoto T., Wadati M., Asymmetric simple exclusion process with open boundaries and Askey-Wilson polynomials, J. Phys. A: Math. Gen. 37 (2004), 4985-5002, cond-mat/0312457.

[23] Arndt P.F., Heinzel T., Rittenberg V., Stochastic models on a ring and quadratic algebras. The three-species diffusion problem, J. Phys. A: Math. Gen. 31 (1998), 833-843, cond-mat/9703182.

[24] Stinchcombe R.B., Schütz G.M., Application of operator algebras to stochastic dynamics and the Heisenberg chain, Phys. Rev. Lett. 75 (1995), 140-143.

[25] Aneva B., Matrix-product ansatz as a tridiagonal algebra, J. Phys. A: Math. Theor. 40 (2007), 11677-11695.

[26] Koornwinder T., The relationship between Zhedanov's algebra $A W(3)$ and the double affine Hecke algebra in the rank one case, SIGMA 3 (2007), 063, 15 pages, math.QA/0612730.

[27] Zhedanov A.S., "Hidden symmetry" of Askey-Wilson polynomials, Teoret. Mat. Fiz. 89 (1991), $190-204$.

[28] Granovskii Y.A., Zhedanov A.S., Linear covariance algebra for SL $L_{q}(2)$, J. Phys. A: Math. Gen. 26 (1993), L357-L359.

[29] Terwilliger P., An algebraic approach to the Askey scheme of orthogonal polynomials, Lecture Notes in Math., Vol. 1883, Editors F. Marcellan and W.V. Assche, Springer, Berlin, 2006, 225-330.

[30] Terwilliger P., Two relations that generalize the $q$-Serre relations and the Dolan-Grady relations, Proceedings of the Nagoya 1999 International Workshop on Physics and Combinatorics, Editors A.N. Kirillov, A. Tsuchiya and H. Umemura, World Sci. Publ., River Edge, NJ, 2001, 377-398, math.QA/0307016.

[31] Dolan L., Grady M., Conserved charges from self-duality, Phys. Rev. D (3) 25 (1982), 1587-1604.

[32] Grabowski M.P., Mathieu P., Quantum integrals of motion for the Heisenberg spin chain, Modern Phys. Lett. A 9 (1994), 2197-2206, hep-th/9403149.

[33] Jimbo M., Miwa T., Algebraic analysis of solvable lattice models, CBMS Regional Conference Series in Mathematics, Vol. 85, American Mathematical Society, Providence, RI, 1995.

[34] Koekoek R., Swarttouw R.F., The Askey-scheme of hypergeometric orthogonal polynomials and its $q$ analogue, Report 98-17, Faculty of Technical Mathematics and Informatics, Delft University of Technology, 1998, math.CA/9602214.

[35] Gasper G., Rahman M., Basic hypergeometric series, Encyclopedia of Mathematics and Its Applications, Vol. 35, Cambridge University Press, Cambridge, 1990.

[36] de Gier J., Essler F.H., Exact spectral gaps of the asymmetric simple exclusion process with open boundaries, J. Stat. Mech. (2006), P12011, 45 pages, cond-mat/0609645. 